\title{
Risk Analysis of the Stock Price Index of Countries Participating in the "Belt and Road" Initiative - Based on GARCH-VaR Model
}

\author{
Maoguo $\mathrm{Wu}^{1} \&$ Daimin $\mathrm{Lu}^{1}$ \\ ${ }^{1}$ SHU-UTS SILC Business School, Shanghai University, Shanghai, China \\ Correspondence: Maoguo Wu, SHU-UTS SILC Business School, Shanghai University, Shanghai, China.
}

Received: February 21, 2019

Accepted: March 9, 2019

Online Published: March 13, 2019

doi:10.5430/ijfr.v10n2p61

URL: https://doi.org/10.5430/ijfr.v10n2p61

\begin{abstract}
The "Belt and Road" Initiative has attracted worldwide attention since its initial stage. The initiative is to unite countries participating in the "Belt and Road" Initiative (B\&R countries), to build a community with a shared future for mankind, and to achieve mutual benefit and win-win. Since the implementation of the initiative, China's outward foreign direct investment (OFDI) has ushered in a new upsurge, and a large amount of money has been invested in B\&R countries. However, China lacks experience in OFDI, as it has not been long since China engaged in OFDI. Besides, most of the $\mathrm{B} \& \mathrm{R}$ countries are developing countries with immature market. As the barometer of the macroeconomy, the stock market can reflect fluctuations of the real economy and forecast the development trend of the macroeconomy. To explore the opportunities and challenges brought by the "Belt and Road" Initiative to the stock market of B\&R countries, this study selects 8 countries with the most active stock market among $B \& R$ countries, and analyzes the impact of the "Belt and Road" Initiative on the stock price index risk of the 8 countries. In this study, the data are divided into 2 groups, i.e., pre-initiative and post-initiative. The GARCH-VaR model is used to calculate the stock price index risk of each country. The empirical results show that the "Belt and Road" Initiative has different effects on the stock price index risk of the 8 countries. After the "Belt and Road" Initiative, the fluctuation of China Shanghai Shenzhen 300 Stock Index Futures is far lower than that before the implementation of the initiative, and the stock price index risk of some countries has also been reduced.
\end{abstract}

Keywords: the "Belt and Road" Initiative, stock price index risk, GARCH-VaR

\section{Introduction}

Nowadays, the world pattern is undergoing complex changes, and international competition is becoming increasingly fierce. How to strengthen cooperation among countries and achieve mutual benefit and win-win situation has become an important issue. The "Belt and Road" Initiative has opened up new ideas for development, and one of its purposes is to build a common-interest community of economic integration. Since the implementation of the initiative, China's outward foreign direct investment (OFDI) has ushered in a new upsurge, with a large amount of capital invested in countries participating in the "Belt and Road" Initiative (B\&R countries). However, China lacks experience in OFDI, as it has not been long since China engaged in OFDI. Besides, most of the B\&R countries are developing countries with immature market. As the barometer of the macroeconomy, the stock market can reflect reflections of the real economy and forecast the development trend of the macroeconomy, and forecast the development trend of the macroeconomy. To explore the opportunities and challenges brought by the "Belt and Road" Initiative to the stock market of B\&R countries, this study investigates the impact of the "Belt and Road" Initiative on the stock price index risks of $8 \mathrm{~B} \& \mathrm{R}$ countries. Not only can studying the fluctuations of the stock price index of B\&R countries reflect the risks and uncertainties of $B \& R$ countries, but also it can reflect the quality and efficiency of the financial markets of B\&R countries. Moreover, this study evaluates the impact of the "Belt and Road" Initiative on the economies of 8 $\mathrm{B} \& \mathrm{R}$ countries, and explores opportunities and challenges that the initiative brings in time.

This study selects $8 \mathrm{~B} \& \mathrm{R}$ countries with the most active stock market judging by the trading volume. The data of the 8 countries are divided into 16 time series, 2 series for each country, i.e., pre-initiative and post-initiative. This study compares the regression results of GARCH family models, chooses the most suitable regression model for each time series, and calculates the VaR values of the stock price index of the $8 \mathrm{~B} \& \mathrm{R}$ countries before and after the implementation of the "Belt and Road" Initiative. Policy implications are put forward accordingly. 


\section{Related Literature}

The VaR model is widely used in financial risk measurement. Baumol (1952) first applied the VaR model to portfolio selection. Morgan (1996) applied the VaR model to the risk measurement system and described the VaR model as a "market volatility measurement standard". Consigli (2002) studied the thick tail of yield distribution and put forward the mean-VaR selection model. Khindanova et al. (2001) pointed out that the value of VaR can be predicted by stable Pareto distribution. Lei (2008) used the VaR model of normal distribution to analyze the risk of stock market, and found that there was a big error in the model. Therefore, the VaR model under normal distribution was put forward and tested. It was found that the forecast result of the improved model was more accurate.

GARCH family models have also been widely used in previous studies. Engle (1982) proposed the ARCH model, which describes conditional variance by a simple quadratic function of lag value. Bollerslev (1986) extended the whole basic ARCH model and described the conditional variance with the square of the lag value or the impact lag value of the conditional variance itself. Nelson (1991) established the EGARCH model, which further improves the GARCH model in the study of leverage effect. Xu (2008) used the GARCH-VaR model to forecast and analyze the risk management and risk level of Hang Seng Index and Shanghai Composite Index.

Despite the large amount of studies and applications based on the VaR model and GARCH family models, there is limited research on the financial market risks of individual countries, especially on the impact of China's development plan on international financial market risks. This study investigates the influence of the "Belt and Road" Initiative on the stock index price risks of B\&R countries. Based on the stock price index of 8 countries, the GARCH-VaR model is used to measure the fluctuations of stock index prices before and after the "Belt and Road" Initiative.

\section{Empirical Analysis}

\subsection{The Data}

This paper selects the stock price index of 8 B\&R countries: China (CSI 300), Russia (RTS), Singapore (STI), India (SENSEX), Poland (WIG), the United Arab Emirates (ADX), Mongolia (MSETOP), and Kazakhstan (KASE). In order to explore the impact of the "Belt and Road" Initiative on these countries' financial market risks, this study divides the stock price index data of each country into two parts: before the "Belt and Road" Initiative (January $1^{\text {st }}$, 2012 - March 27 $7^{\text {th }}, 2015$ ) and after the "Belt and Road" Initiative (March 28 $8^{\text {th }}, 2015$ - January 31 $1^{\text {st }}, 2018$ ), and calculates the VaR value, respectively

This study calculates the profit and loss value of a day's trading using the price $P_{t}$ of the stock index of the 8 countries and the price $P_{t-1}$ of the stock index of the previous trading day. The formula is as follows:

$$
r=\ln P_{t}-\ln P_{t-1}
$$

\subsection{Stock Price Index Risks Before the Initiative}

First, descriptive statistics, normal distribution test, and stationary test (ADF unit root test) of the stock index profit and loss data of the 8 countries are carried out. The results are shown in Table 1.

Table 1. Descriptive statistics, normal distribution test, and ADF unit root test

\begin{tabular}{lllllllll}
\hline Stock Index & Std. Dev. & Skewness & Kurtosis & Jarque-Bera & p-value & ADF value & \multicolumn{2}{c}{-value } \\
\hline CSI 300 & 0.018785 & 0.183923 & 6.092433 & 266.3033 & 0.00 & -15.58528 & 0.00 & Yes \\
\hline RTS & 0.026247 & 1.394301 & 19.88188 & 9893.328 & 0.00 & -15.91571 & 0.00 & Yes \\
\hline STI & 0.008978 & 0.123743 & 3.536836 & 11.80823 & 0.00 & -17.96364 & 0.00 & Yes \\
\hline SENSEX & 0.012887 & 0.028132 & 4.001242 & 33.60548 & 0.00 & -17.56093 & 0.00 & Yes \\
\hline WIG & 0.012217 & 0.476224 & 7.169269 & 613.4759 & 0.00 & -17.96934 & 0.00 & Yes \\
\hline ADX & 0.01359 & 1.013112 & 15.35218 & 5288.013 & 0.00 & -14.70078 & 0.00 & Yes \\
\hline MSETOP & 0.014032 & -0.497358 & 8.931327 & 378.279 & 0.00 & -12.05125 & 0.00 & Yes \\
\hline KASE & 0.022218 & -0.291902 & 9.573965 & 255.9023 & 0.00 & -8.851049 & 0.00 & Yes
\end{tabular}


Jarque-Bera tests find that the stock index profit and loss values of the 8 countries are very large, and the kurtosis value is not 3, i.e., all time series do not conform to normal distribution. In general, the volatility and dispersion of stock index profit and loss values of the 8 countries are not very different, and the smallest dispersion is Singapore STI stock index. In terms of stationarity, before the "Belt and Road" Initiative, the p-values of the ADF unite root tests on the stock index profit and loss values of the 8 countries are all close to 0 , i.e., each time series are stationary, and there is no unit root.

Then, this study establishes an autoregression equation for the profit and loss values of the 8 countries before the "Belt and Road" Initiative. The regression equation is as follows.

$$
\mathrm{r}=\beta_{0}+\beta_{1} \mathrm{r}(-1)+\varepsilon
$$

First, LM serial correlation test is carried out, and the lag of each serial is used to observe whether there is serial correlation in each time series. If serial correlation exists, the Cochrane-Ocutter ARMA model is used to eliminate it. The test results show that the corresponding p-values of LM serial correlation test for each time series are large, rejecting the null hypothesis that serial correlation exists, and there is no serial correlation for each time series.

Then the ARCH-LM method is used to test the ARCH effect of each time series. In 7 countries, the corresponding p-values of the 1 order lag of the stock index profit and loss values are significantly lower than the $1 \%$ significance level, while observations of the Kazakhstan KASE index profit and loss value is scanty before the "Belt and Road" Initiative, but the p-value of the 1 order lag of the stock index profit and loss value is still less than the 5\% significance level. In addition, the F-values of the ARCH-LM test on the stock index profit and loss values of the 8 countries are very large, so it rejects the null hypothesis that ARCH effect exists.

Afterwards, the profit and loss values of the 8 stock indexes are substituted into GARCH/TARCH model, EGARCH model, and PARCH model, respectively. According to the regression results, the most suitable regression model for each time series is selected, i.e., the model with the highest goodness of fit (the largest $R^{2}$ ). Formulas of the GARCH family model are as follows.

GARCH/ TARCH Model:

$$
\sigma_{t}^{2}=\alpha_{0}+\sum_{i=1}^{p} \alpha_{i} r_{t-i}^{2}+\sum_{j=1}^{\alpha} \alpha_{j} \sigma_{t-j}^{2}
$$

EGARCH Model:

$$
\begin{gathered}
\log \sigma_{t}^{2}=\alpha+\sum_{k=1}^{\infty} \beta_{k} * g *\left(z_{t-k}\right)+\sum_{j=1}^{q} \alpha_{j} * \log \left(\sigma_{t-j}^{2}\right) \\
g\left(z_{t}\right)=\theta_{z_{t}}+\gamma\left[\left|z_{t}\right|-E\left|z_{t}\right|\right]
\end{gathered}
$$

PARCH Model:

$$
\sigma_{t}^{\delta}=\alpha\left(\left|\varepsilon_{t-1}\right|\right)^{\delta}+\beta \sigma_{t-1}^{\delta}
$$

The regression results of GARCH family models and the selection of optimal models are shown in Table 2 .

Table 2. Regression results of GARCH family models and selection of optimal models

\begin{tabular}{llllll}
\hline & & CSI 300 & RTS & STI & SENSEX \\
\hline GARCH & $R^{2}$ & 0.229624 & $\mathbf{0 . 1 8 8 8 3 0}$ & $\mathbf{0 . 2 7 6 5 0 8}$ & $\mathbf{0 . 1 8 7 6 0 9}$ \\
\hline PARCH & $R^{2}$ & $\mathbf{0 . 2 2 9 8 2 5}$ & 0.187860 & 0.275327 & 0.187127 \\
\hline EGARCH & $R^{2}$ & 0.229085 & 0.185935 & 0.272873 & 0.185834 \\
\hline \multicolumn{2}{l}{ Optimal Model } & PARCH & GARCH & GARCH & GARCH \\
\hline \multicolumn{2}{l}{ GIG } & ADX & MSETOP & KASE \\
\hline GARCH & $R^{2}$ & 0.238903 & 0.203734 & $\mathbf{0 . 0 6 8 0 3 9}$ & 0.187609 \\
\hline EARCH & $R^{2}$ & $\mathbf{0 . 2 3 8 3 3 9}$ & $\mathbf{0 . 2 0 4 0 4 2}$ & 0.043727 & 0.187127 \\
\hline Optimal Model & $R^{2}$ & 0.238873 & 0.204256 & 0.051770 & $\mathbf{0 . 1 8 5 8 3 4}$ \\
\hline
\end{tabular}

Before analyzing the regression results of GARCH family models, this study re-examines the ARCH effect of each 
time series, and observes whether ARCH effect still exists in the residual series of each group of data regression. If there is no ARCH effect, it shows that the regression results of GARCH family models are effective. The re-test results of ARCH effect are shown in Table 3.

Table 3. ARCH effect re-test

\begin{tabular}{lllllllll}
\hline & CSI 300 & RTS & STI & SENSEX & WIG & ADX & MSETOP & KASE \\
\hline F-value & 0.6787 & 1.3895 & 1.8031 & 0.0340 & 0.5035 & 0.0014 & 0.2710 & 0.1664 \\
\hline p-value & 0.4103 & 0.2388 & 0.1797 & 0.8537 & 0.1140 & 0.9697 & 0.6031 & 0.6840 \\
\hline
\end{tabular}

The test results show that there is no ARCH effect in the residual series of data regression, and the GARCH family models are effective.

After the GARCH family models are re-tested by ARCH effect, the VaR values corresponding to each time series are calculated. This study chooses the static prediction which is more suitable for the data in this study. According to the student's t-value of the regression results of GARCH family models, the quantile critical values corresponding to the 95\% confidence level are calculated. The VaR values of the 8 stock indexes are predicted by the VaR calculation formula, as shown in Table 4.

Table 4. Prediction results of VaR values

\begin{tabular}{llllllll}
\hline \multirow{2}{*}{$\begin{array}{l}\text { Quantile } \\
\text { Critical Values }\end{array}$} & \multirow{2}{*}{ Student's T } & VaR & & & \\
\cline { 5 - 8 } & & & Mean & Medium & Maximum & Minimum & Std. Dev. \\
\hline CSI 300 & 8.010779 & 1.859224 & -0.029730 & -0.026410 & -0.008670 & -0.128516 & 0.011756 \\
\hline RTS & 6.033060 & 1.941275 & -0.041784 & -0.036034 & -0.010665 & -0.266148 & 0.021454 \\
\hline STI & 29.66922 & 1.697864 & -0.012648 & -0.011594 & -0.001638 & -0.038015 & 0.005519 \\
\hline SENSEX & 15.84890 & 1.746905 & -0.019808 & -0.018447 & -0.004777 & -0.063073 & 0.007160 \\
\hline WIG & 15.51521 & 1.749236 & -0.018516 & -0.016641 & -0.009054 & -0.086661 & 0.007376 \\
\hline ADX & 4.420587 & 2.074884 & -0.022740 & -0.018203 & -0.005925 & -0.172759 & 0.016722 \\
\hline MSETOP & 3.928187 & 2.143094 & -0.025666 & -0.021632 & -0.012638 & -0.081987 & 0.011989 \\
\hline KASE & 4.644979 & 2.049573 & -0.038487 & -0.029427 & -0.011432 & -0.138831 & 0.025270 \\
\hline
\end{tabular}

In order to ensure the validity of the predicted VaR values, this study carries out chi-square test on the predicted VaR values and calculates the chi-square critical value at the $95 \%$ confidence level. The results are shown in Table 5 .

Table 5. Chi-Square tests of predicted var values

\begin{tabular}{llllllll}
\hline CSI 300 & RTS & STI & SENSEX & WIG & ADX & MSETOP & KASE \\
\hline 6606.52 & 1819.04 & 5679.34 & 8416.46 & 6058.58 & 353.35 & 472.88 & 84.86 \\
\hline
\end{tabular}

The results show that the chi-square values corresponding to all predicted VaR values are much larger than the chi-square critical values at the $95 \%$ confidence level, i.e., the VaR values conform to the chi-square distribution, and the predicted values are effective.

\subsection{Stock Price Index Risks After the Initiative}

In this study, the data processing method of Section 3.2 is applied to analyze the data after the "Belt and Road" Initiative. Descriptive statistics, normal distribution test, and stationary test (ADF unit root test) are shown in Table 6. 
Table 6. Descriptive statistics, normal distribution test, and ADF unit root test

\begin{tabular}{lllllllll}
\hline Stock Index & Std. Dev. & Skewness & Kurtosis & Jarque-Bera & p-value & ADF value & p-value & Stationary \\
\hline CSI 300 & 0.022812 & 0.484415 & 8.448767 & 890.7553 & 0.00 & -11.95373 & 0.00 & Yes \\
\hline RTS & 0.022277 & 0.044679 & 4.612967 & 77.85452 & 0.00 & -15.92722 & 0.00 & Yes \\
\hline STI & 0.010432 & 0.136432 & 5.733541 & 226.0866 & 0.00 & -17.19915 & 0.00 & Yes \\
\hline SENSEX & 0.011569 & 0.294464 & 5.906185 & 257.9207 & 0.00 & -14.88829 & 0.00 & Yes \\
\hline WIG & 0.012114 & 0.155481 & 4.845994 & 103.9636 & 0.00 & -13.51129 & 0.00 & Yes \\
\hline ADX & 0.012331 & -0.076735 & 5.388238 & 169.6692 & 0.00 & -16.51075 & 0.00 & Yes \\
\hline MSETOP & 0.015367 & 0.248496 & 12.53546 & 2689.576 & 0.00 & -19.31632 & 0.00 & Yes \\
\hline KASE & 0.014795 & -0.024189 & 6.261494 & 308.9942 & 0.00 & -13.91368 & 0.00 & Yes \\
\hline
\end{tabular}

Table 6 shows that all time series do not conform to normal distribution. ADF unit root tests show that the profit and loss values of the 8 countries after the "Belt and Road" Initiative are stationary and there is no unit root.

The same autoregression equation as in Section 3.2 is established for the 8 stock index profit and loss values. LM serial correlation tests show that there is no serial correlation in each time series. ARCH-LM tests show that there is ARCH effect in each time series.

The 8 stock index profit and loss values are substituted into GARCH/TARCH model, EGARCH model, and PARCH model, respectively. According to the regression results, the most suitable regression model for each time series is selected, i.e., the model with the highest goodness of fit (the largest $R^{2}$ ). The regression results of GARCH family models and the selection of optimal models are shown in Table 7.

Table 7. Regression results of GARCH family models and selection of optimal models

\begin{tabular}{|c|c|c|c|c|c|}
\hline & & CSI 300 & RTS & STI & SENSEX \\
\hline GARCH & $R^{2}$ & 0.170660 & 0.176675 & 0.192246 & 0.206515 \\
\hline PARCH & $R^{2}$ & 0.170279 & 0.176020 & 0.192430 & 0.202150 \\
\hline EGARCH & $R^{2}$ & 0.170145 & 0.175843 & 0.192500 & 0.201507 \\
\hline \multicolumn{2}{|c|}{ Optimal Model } & PARCH & GARCH & GARCH & GARCH \\
\hline & & WIG & ADX & MSETOP & KASE \\
\hline GARCH & $R^{2}$ & 0.170457 & 0.242141 & 0.165689 & 0.152040 \\
\hline PARCH & $R^{2}$ & 0.168749 & 0.241800 & 0.167058 & 0.152409 \\
\hline EGARCH & $R^{2}$ & 0.168860 & 0.242222 & 0.166150 & 0.150728 \\
\hline Optimal N & & PARCH & GARCH & EGARCH & PARCH \\
\hline
\end{tabular}

The results of ARCH effect re-test show that there is no ARCH effect in the residual series of data regression, and GARCH family models are effective. The results of ARCH effect re-test are detailed in Table 8.

Table 8. ARCH effect re-test

\begin{tabular}{|c|c|c|c|c|c|c|c|c|}
\hline & CSI 300 & RTS & STI & SENSEX & WIG & ADX & MSETOP & KASE \\
\hline F-value & 0.6251 & 1.5612 & 1.8613 & 0.0362 & 0.5713 & 0.0021 & 0.2823 & 0.1724 \\
\hline p-value & 0.4415 & 0.2516 & 0.1801 & 0.8612 & 0.1233 & 0.9741 & 0.6718 & 0.6913 \\
\hline
\end{tabular}


After GARCH family models are re-tested for ARCH effect, the VaR values corresponding to each time series are calculated. This study chooses the static prediction which is more suitable for the data in this study. According to the student's t-value of the regression results of GARCH family models, the quantile critical value corresponding to the 95\% confidence level is calculated. The VaR values of the 8 stock indexes are predicted by the VaR calculation formula, as shown in Table 9.

Table 9. Prediction results of VaR values

\begin{tabular}{llllllll}
\hline & \multirow{2}{*}{$\begin{array}{l}\text { Quantile } \\
\text { Critical } \\
\text { Values }\end{array}$} & Student's T & & \multicolumn{2}{l}{ VaR } \\
\cline { 5 - 8 } & & & Mean & Medium & Maximum & Minimum & Std. Dev. \\
\hline CSI 300 & 7.756194 & 1.867154 & -0.031673 & -0.021664 & -0.004256 & -0.185642 & 0.025904 \\
\hline RTS & 13.31081 & 1.767777 & -0.033742 & -0.030626 & -0.007746 & -0.108041 & 0.015814 \\
\hline STI & 11.53592 & 1.788281 & -0.015900 & -0.014229 & -0.003481 & -0.059065 & 0.006780 \\
\hline SENSEX & 17.19905 & 1.738449 & -0.017192 & -0.015306 & -0.001020 & -0.076298 & 0.007524 \\
\hline WIG & 13.78230 & 1.763278 & -0.019094 & -0.017871 & -0.005876 & -0.070861 & 0.006703 \\
\hline ADX & 6.564780 & 1.913647 & -0.019507 & -0.017370 & -0.005233 & -0.071298 & 0.009736 \\
\hline MSETOP & 5.265027 & 1.992857 & -0.025495 & -0.022675 & -0.014630 & -0.185308 & 0.012468 \\
\hline KASE & 8.839544 & 1.676955 & -0.021563 & -0.018923 & -0.010303 & -0.135549 & 0.010826 \\
\hline
\end{tabular}

In order to ensure the validity of the predicted values of VaR, this study carries out chi-square test on the predicted values of $\mathrm{VaR}$ and calculates the chi-square critical value at the $95 \%$ confidence level. The results show that the chi-square values corresponding to all predicted values of $\mathrm{VaR}$ are much larger than the chi-square critical values at the $95 \%$ confidence level, i.e., the VaR values conform to the chi-square distribution, and the predicted values are effective.

Comparing the VaR values of the stock price index of the 8 countries before and after the "Belt and Road" Initiative, it can be found that after the implementation of the "Belt and Road" Initiative, China CSI 300, Russia RTS, Singapore STI, India SENSEX, Poland WIG, and Kazakhstan KASE all exhibited more volatile at the initial stage, and the volatility of risk value tended to be stable in the late 2016. The later value volatility of China CSI 300 is much lower than that before the implementation of the "Belt and Road" Initiative. At the same time, the volatility of risk value of Mongolia MSETOP and Kazakhstan KASE decreased significantly compared with that before the implementation of the "Belt and Road" Initiative, but the volatility of risk value of UAE ADX increased significantly.

\section{Conclusion and Policy Implications}

The "Belt and Road" Initiative, which was put forward in September 2013 and officially launched in March 2015, has been the focus of attention both at home and abroad. Since the implementation of the initiative, China's OFDI has been increasing, bringing opportunities for economic development to B\&R countries. However, the "Belt and Road" Initiative faces many challenges in its implementation. This study selects stock index data from 8 countries (China, Russia, Singapore, India, Poland, the United Arab Emirates, Mongolia, and Kazakhstan) from January $1^{\text {st }}$, 2012 to January $31^{\text {st }}, 2018$, taking March 2015 as the node. This study utilizes the GARCH-VaR model to measure the stock price index of the 8 countries before and after the initiative, and evaluates the impact of the "Belt and Road" plan on the stock price index risk of the 8 countries.

The applicability of GARCH models to the profit and loss values of stock indexes in the 8 countries is different. This study chooses the regression model which is most suitable for each time series, and calculates the VaR values of the stock price index of each country before and after the initiative. The empirical results show that compared with the volatility before the implementation of the "Belt and Road" Initiative, the volatility of the risk value of China CSI 300 is significantly smaller, while the volatility of the risk value of Russia RTS, Singapore STI, India SENSEX, Poland WIG, and UAE ADX has increased. In addition, the volatility of the risk value of Mongolia MSETOP and Kazakhstan KASE is smaller than that of other countries. China CSI 300, Russia RTS, Singapore STI, India 
SENSEX, Poland WIG, and Kazakhstan KASE exhibited more volatility in the early stage after the implementation of the "Belt and Road" Initiative, but the volatility of value at risk in the late 2016 tended to be stable, especially for CSI China 300. The volatility of value at risk was much lower than that before the implementation of the "Belt and Road" Initiative. The volatility of value at risk of Mongolia MSETOP and Kazakhstan KASE is much more stable than that before the "Belt and Road" Initiative. However, the volatility of value at risk of UAE ADX is much greater than before.

The results show that the "Belt and Road" Initiative reduces the risk of stock price index of some B\&R countries, but there is no change in the stock price index risk of several countries. The possible reasons are: firstly, the "Belt and Road" Initiative is still in its infancy. Many policies have been put forward but have not yet been implemented. Secondly, China is a developing country and its economic influence needs to be improved. Thirdly, the implementation of the "Belt and Road" Initiative is largely restricted by the national conditions and economic level of $\mathrm{B} \& \mathrm{R}$ countries. Therefore, this study proposes a series of corresponding policy implications for the above three problems: firstly, the government should advance the "Belt and Road" Initiative step by step instead of hastily. The initiative should also be considered in the long run. Secondly, the government should actively promote the "Belt and Road" Initiative at the level of diplomacy and publicity so as to enhance the international visibility of the initiative and promote the participation of neighboring countries. Thirdly, measures should be taken according to local conditions to avoid potential risks of $\mathrm{B} \& \mathrm{R}$ countries (such as war, epidemics, short-term economic slump etc.), understand and fully respect the national conditions of the destination countries, strive to maximize the interests of both sides, and work together for development.

As a major international policy, the "Belt and Road" Initiative is bound to be a very important reference for investors and a relatively positive news. However, at present, the "Belt and Road" Initiative is only at the initial stage, and it can only play a positive role in some Chinese multinational companies at present. For small and medium-sized investors, they should not blindly rely on the good news of the "Belt and Road" Initiative in the international market. They should also weigh the situation of China and weigh the situation of other countries and make rational judgments. The impact of the "Belt and Road" Initiative on China's market economy is not comprehensive. Small investors should carefully study the breadth and depth of the "Belt and Road" Initiative on the coverage of companies. They should also fully investigate the background of companies and formulate decisions accordingly.

\section{References}

Baumol, W. J. (1952). The Transactions Demand for Cash: An Inventory Theoretic Approach. Quantitative Journal of Economy, 66(4), 545-555. https://doi.org/10.2307/1882104

Bollerslev, T. (1986). Generalized Autoregressive Conditional Heteroscedasticity. Journal of Econometrics, 31(6), 307-327. https://doi.org/10.1016/0304-4076(86)90063-1

Consigli, G. (2002). Tail Estimation and Mean-VaR Portfolio Selection in Markets Subject to Financial Instability. Journal of Banking and Finance, 26, 1355-1382. https://doi.org/10.1016/S0378-4266(02)00267-4

Engle, R. F. (1982). Autoregressive Conditional Heteroscedasticity with Estimates of the Variance of United Kingdom Inflation. Econometrica, 50, 987-1008. https://doi.org/10.2307/1912773

Khindanova, I. (2001). Stable Modeling of Value at Risk. Mathematical and Computer Modeling, (34). https://doi.org/10.1016/S0895-7177(01)00129-7

Lei, X. (2008). VaR under Stable Distribution. Master Dissertation, Jinan University.

Morgan, J. P. (1996, November/December). Measuring the Risk in Value at Risk. Financial Analysis Journal, 47-55.

Nelson, D. (1991). Conditional Heteroskedasticity in Asset Returns: A New Approach. Econometrica, 347-370. https://doi.org/10.2307/2938260

$\mathrm{Xu}, \mathrm{L}$. (2008). Risk Measurement and Management Strategy of Stock Index Futures Operation. Statistics and Decisions, (21), 118-120. 